\title{
Smartphone-based heart-rate measurement using facial images and a spatiotemporal alpha-trimmed mean filter
}

\author{
J.-S. Lee*, K.-W. Lin and J.-L. Syue \\ Department of Computer Science and Information Engineering, National University of Tainan, Tainan, \\ Taiwan
}

\begin{abstract}
Currently, cardiovascular disease affects a relatively high proportion of the world's population. Thus, developing simple and effective methods for monitoring patients with cardiovascular disease is critical for research. Monitoring the heart rate of patients is a relatively simple and effective method for managing patients with this condition. For patients, the desired heart rate monitoring equipment should be portable, instantaneous, and accurate. Because smartphones have become the most prevalent mobile device, we utilized this technology as a platform for developing a novel heart-rate measurement system. Catering to the phenomenon of people using the front camera of their smartphones as a mirror, the proposed system was designed to analyze facial-image sequences captured using the front camera. A spatiotemporal alpha-trimmed mean filter was developed to estimate a user's heart rate quickly and accurately. The experimental results show that in addition to achieving these objectives, the developed system outperforms a similar personal computer-based system. In addition, the system performs effectively even when users are wearing glasses. Hence, the proposed system demonstrates practical value for people who must monitor their heart rate daily.
\end{abstract}

Keywords: Smartphone, photoplethysmography (PPG), heart rate

\section{Introduction}

Currently, the global prevalence of cardiovascular disease is relatively high. Thus, developing methods that enable patients to monitor their condition easily and effectively has become a prominent research topic $[1,2]$. Monitoring the heart rate of patients with cardiovascular disease is a relatively simple and effective method for managing their condition. Heart-rate variability has been adopted as an indicator for predicting myocardial infarction [3] and liver cancer [4]. The most common procedure for measuring the heart rate is an electrocardiogram (ECG). However, ECG measurements are typically performed by professionals in hospitals or health care institutions. These constraints are inconvenient for people who must monitor their heart rate regularly. In addition, more than $20 \%$ of hypertensive patients experience the white-coat effect in a hospital setting [5], which can cause inaccurate heart-rate measurements. Although portable electrocardiographs can be employed to continuously record a patient's heart rate for 24 consecutive hours, analyzing the data can be time-consuming.

\footnotetext{
${ }^{*}$ Corresponding author: J.-S. Lee, Department of Computer Science and Information Engineering, National University of Tainan, 33, Sec. 2, Shu-Lin St. Tainan, Taiwan.E-mail: cslee@mail.nutn.edu.tw.
} 
A plethysmograph is an instrument for measuring changes in blood volume [6-9]. Compared with other heart-rate monitoring techniques, photoplethysmography (PPG) [8] is superior because of the portability of the involved instruments. PPG is typically implemented using a specific light source (e.g. red or infrared wavelengths), and it is generally attached to measuring sites (e.g., the fingertips or earlobes) in a manner that can cause patients to feel uncomfortable. Pulse measurements can be acquired using digital cameras under normal ambient light conditions, indicating that the heart rate can be evaluated by contactless periodic variation measurement of reflectance strength resulting from varying hemoglobin absorptivity across the visible light spectrum as blood volume in the blood vessels increases and decreases with every heartbeat.

Poh et al. [10] presented a method that involves using a webcam for contactless cardiac pulse measurement based on blind-source separation. The results indicated that optimal performance can be obtained by estimating the heart rate directly from a green-channel intensity image. Chen et al. [11] proposed a framework for decomposing brow reflectance from the green channel and then decomposing intrinsic mode functions by using ensemble empirical mode decomposition to separate the effect component that represents the real heart-rate variation from environmental noise.

However, these two methods are subject to complex computation; hence, they require using a personal computer (PC). To facilitate heart-rate monitoring for people, smartphones are a potentially effective platform. However, because smartphones have comparatively limited computational resources, they are generally unsuitable for such complex algorithms. Therefore, we developed a simple and fast algorithm based on a spatiotemporal alpha-trimmed mean filter (ATMF) to estimate a user's heart rate by capturing a sequence of images of his or her front face through the front camera on their smartphone.

The remainder of this paper is organized as follows. Section 2 describes the proposed method, and Section 3 presents the experimental results and a discussion. Finally, some concluding remarks are offered in Section 4.

\section{The proposed method}

An ideal portable heart-rate measurement instrument should be operated intuitively. Many people groom themselves by using the front camera of their smartphone as a mirror. Hence, the operating mode of the proposed system is similar to grooming. As shown in Fig. 1, operating the system requires the user to position the phone so that his or her entire face is visible on the phone screen; as a guide, the system displays a red box indicating the region of interest (ROI). The system captures a sequence of ROIs, which are then used as input data. Assuming that the phone camera can capture images at a rate of 10 frames per second, $4 \mathrm{~s}$ is sufficient for capturing enough frames to estimate the user's heart rate. In such a short period, the light intensity irradiated to the ROI can be assumed constant. Some of the light is absorbed into skin, whereas some of it is reflected. The amount of reflected light changes over time. Blood volume can be further divided into arterial and venous. Because arterial oxyhemoglobin can absorb light, the reflected light coming from arterial blood changes with the heartbeat. Thus, the heart rate can be estimated by analyzing the reflected light intensity of the ROI over time (Fig. 2).

Poh et al. [10] found that the variation in reflected light caused by the heart beating is most evident in the green component. Therefore, in the proposed system, only the green component of the ROI is extracted for subsequent analysis. The green component sequence $G(t)$ can be obtained by applying Eq. (1). Because the pulse frequency generated by the heart beating is approximately $0.7-3.0 \mathrm{~Hz}$, the system uses a bandpass filter to remove noise components outside the heartbeat frequency band. The filtered signal $B(t)$ is expressed in Eq. (2), where $F(t)$ is the corresponding kernel of the bandpass filter 


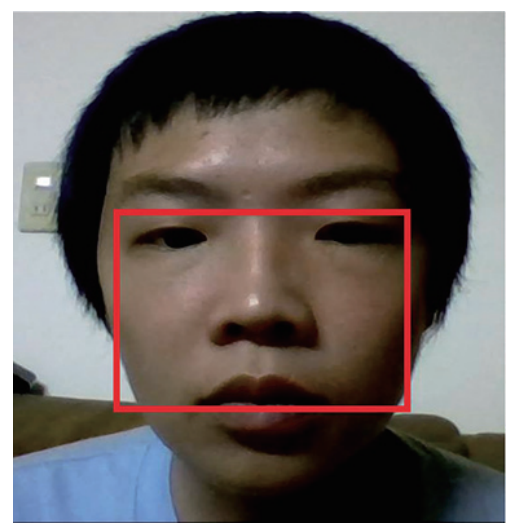

Fig. 1. Red box indicating the ROI.

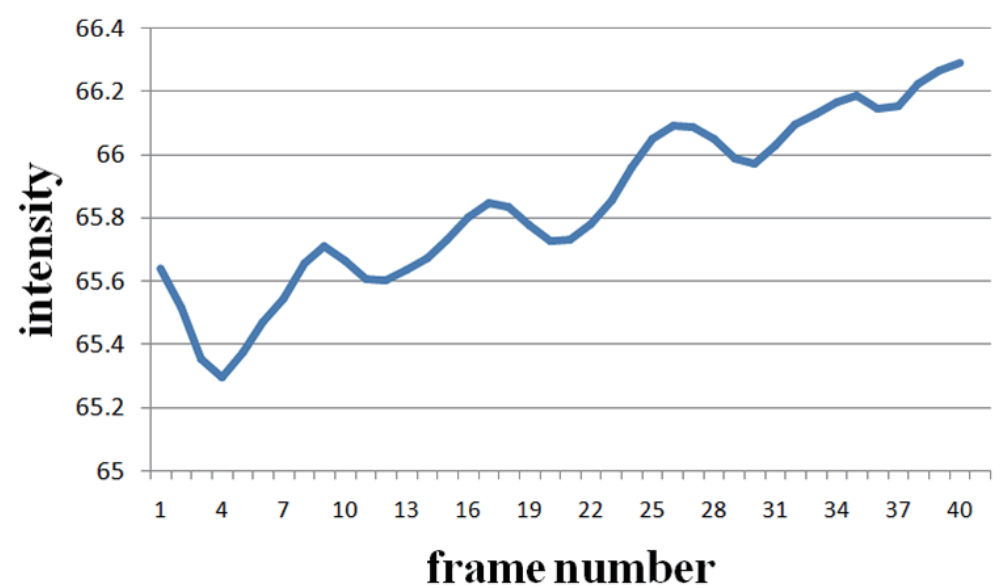

Fig. 2. Reflected green light intensity $G(t)$ in the ROI over time.

and $\otimes$ represents the convolution operator. Subsequently, the corresponding heart rate can be estimated according to an estimate of the peak-to-peak time interval (Fig. 3). Each peak in $B(t)$ corresponds to one heartbeat, and the number of peaks in a time interval represents the heart rate. To detect the peaks in each period, the location of the local maxima is computed using Algorithm 1.

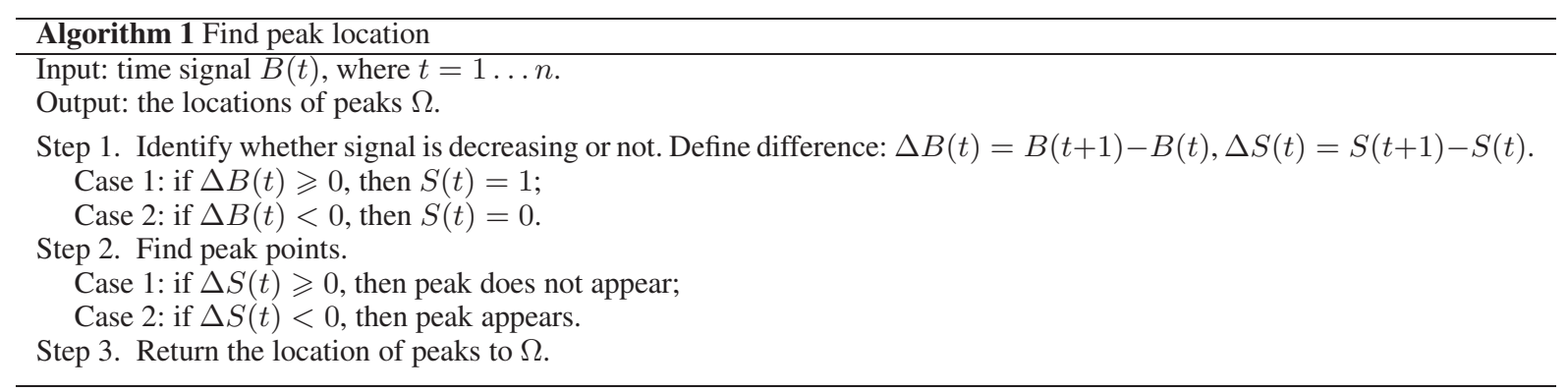

The time interval between the peak $i$ and the peak $(i+1)$ corresponds to the $i$ th heartbeat cycle, denoted as $T_{i}$ (Eq. (3)). The heartbeat cycle sequences are denoted as $\left\{T_{i} \mid i=1,2, \ldots, k\right\}$. Spurious heartbeat 


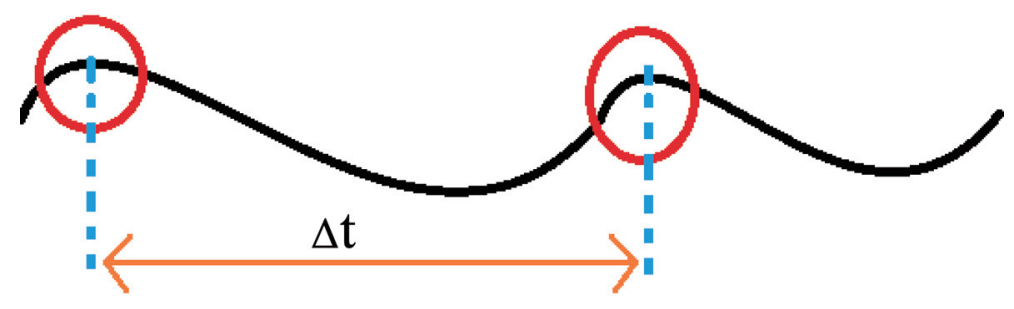

Fig. 3. Schematic diagram depicting the heartbeat cycle.

cycles must be filtered to ensure that they do not affect the accuracy of the estimated heart rate. The system invokes the ATMF, an effective and efficient noise filter, to eliminate spurious heartbeat cycles for ensuring that the acquired heartbeat cycle is representative. For the ATMF, outliers in the data, which generally correspond to extremes in the ranked data, are regarded as noise. The data input into the ATMF must be ranked in ascending order in advance. The sorted results are expressed as $\left\{R T_{i} \mid i=1,2, \ldots, k\right\}$, $R T_{i}<R T_{i+1}$. The ATMF is defined in Eq. (4). The average heart rate $H R$ can be derived using Eq. (5).

The above ATMF is employed to mitigate noise in the temporal domain. To further enhance the accuracy of the estimated heart rate, the ATMF is also employed to mitigate noise in the spatial domain. The noise results from uneven illumination of the face can be reduced by spatial partitioning, which involves dividing the ROI into small blocks to minimize the uneven illuminance in each block. In this study, the ROI is divided into $3 \times 3$ blocks, where the block at row $i$ and column $j$ is denoted as $\operatorname{ROI}(i, j)$. The heart-rate estimation process is repeated for each $\operatorname{ROI}(i, j)$. The corresponding estimated heart rate is denoted as $H R(i, j)$. A series of estimations $\{H R(i, j) \mid i=1,2,3 ; j=1,2,3\}$ are ranked in ascending order, yielding $\left\{R H R_{i} \mid i=1,2, \ldots, 9\right\}, R H R_{i}<R H R_{i+1}$. The final estimated heart rate $\overline{H R}$ is obtained by applying the ATMF in the spatial domain, as expressed in Eq. (6).

$$
\begin{aligned}
& G(t)=\frac{1}{M N} \sum_{j=0}^{N-1} \sum_{i=0}^{M-1} R O I_{G}(i, j, t) \\
& B(t)=F(t) \otimes G(t) \\
& T_{i}=\Omega(i+1)-\Omega(i) \\
& T=\frac{1}{K-2 p} \sum_{i=p}^{K-p} R T_{i} \\
& H R=\frac{60}{T} \\
& \overline{H R}=\frac{1}{9-2 p} \sum_{i=p}^{9-p} R H R_{i}
\end{aligned}
$$



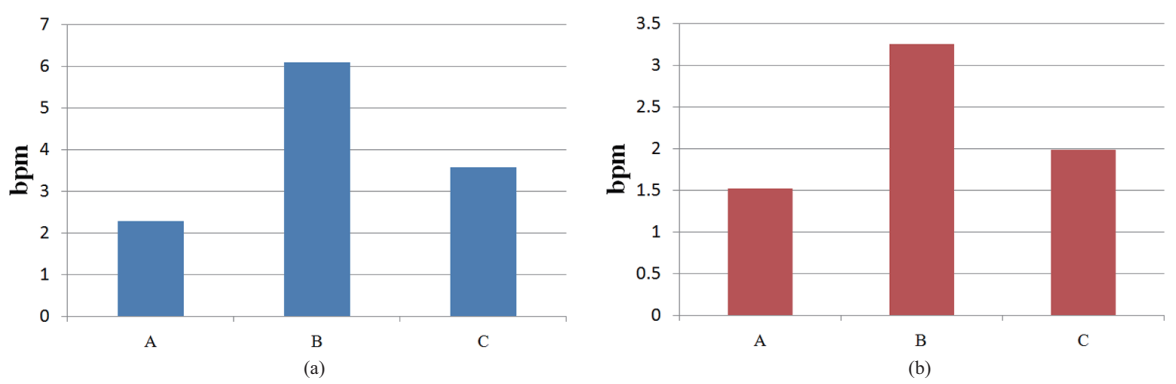

Fig. 4. Error values for Conditions A-C: MAE chart (Fig. 4(a)); SD chart (Fig. 4(b)).

\section{Experimental results}

The proposed system was developed using the Eclipse Android Software Development Kit and tested on a Samsung Galaxy S3 I9300. In the experiments, the ground truth of the heart rate was obtained using a sphygmomanometer (TERUMO ES-P310) following the specification of IEC 60601-1-2:2001 (measurement deviation: $\pm 5 \%$ ). Because ambient light is the most influential variable in PPG-based measurements, we evaluated the proposed system under three ambient light conditions: daytime indoor (Condition A), daytime outdoor (Condition B), and nighttime indoor (Condition C). For Conditions A and $\mathrm{B}$, the light source was natural sunlight, whereas that for Condition $\mathrm{C}$ was a fluorescent lamp. For each condition, six subjects underwent five tests. The error value in each test was obtained by comparing the measurements with the ground truth, Figures 4(a) and 4(b) show the mean absolute error (MAE) and standard deviation (SD), respectively. The results reveal that the optimal performance was achieved under Condition $\mathrm{A}(\mathrm{MAE}=2.28 \mathrm{bpm} ; \mathrm{SD}=1.52 \mathrm{bpm})$. This result also shows that stronger sunlight can generate larger measurement errors. A possible explanation for this result is that the phone camera receives a larger green light component from the background environment compared with the blood reflection. Furthermore, the ambient green light intensity may fluctuate over time. The measurement error under Condition $\mathrm{C}$ may have resulted from the high-frequency flicker of the fluorescent light. Most of the flicker noise can be removed using the bandpass filter to minimize the error values. Condition $\mathrm{A}$ is the ideal condition because the interference is minimal. In addition, the system response time is a crucial determinant of user acceptance. The proposed system requires merely $4 \mathrm{~s}$ to capture sufficient input data, and only $3.6 \mathrm{~s}$ to estimate the heart rate. The agile response is due to the highly efficient algorithm incorporated into the system.

The proposed system captures the entire face for measurement. To test whether wearing glasses influences the results, we conducted a second experiment, for which the subjects wore glasses under Condition $\mathrm{A}$. The error values (MAE $=3.24 \mathrm{bpm} ; \mathrm{SD}=2.95 \mathrm{bpm}$ ) show that although wearing glasses affects the estimation accuracy, the influence is negligible.

To understand the relative effectiveness of the proposed system in heart-rate estimation, we used the system proposed in [11] as a comparison target. This system was compared because the same equipment was used to obtain the ground truth. Furthermore, because a Bland-Altman plot rather was adopted in [11], we used a Bland-Altman plot to compare the performance. The test conditions in [11] were identical to Condition A; therefore, we performed the tests under Condition A to achieve an objective comparison. Figures 5(a) and 5(b) show the results of the second experiment via Bland-Altman plots. In [11], the error variation range was approximately $17 \mathrm{bpm}$, and our margin of error was approximately $13 \mathrm{bpm}$. These results show that in addition to operating as intended, the system proposed in the present study outperformed the PC-based platform, despite the limited computational resources of the mobile phone. 


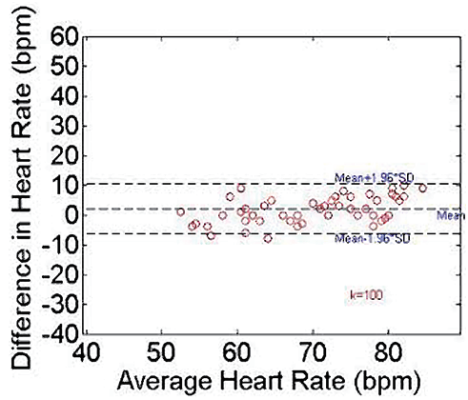

(a)

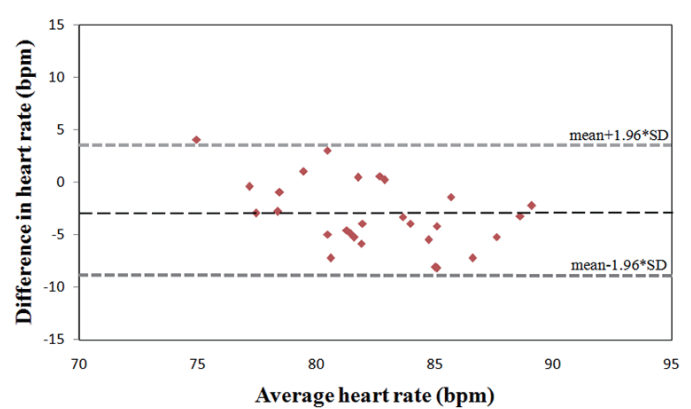

(b)

Fig. 5. Bland-Altman plots depicting the distribution of the testing samples: obtained from [11] (Fig. 5(a)); obtained using the proposed system (Fig. 5(b)).

\section{Conclusion}

In this study, we used a smartphone as a platform to develop a personal heart-rate measurement system for assisting people in managing their health. To operate the proposed system, smartphone users capture a sequence of facial images by using the front camera on their smartphone. The time-series data of the green component are input into a bandpass filter to remove signals with no cardiac component. Subsequently, the signal peaks are detected to derive the cardiac cycle series. Finally, the heart rate is estimated using the proposed spatiotemporal ATMF. The experimental results show that the optimal estimation accuracy is achieved when the measurements are performed in an indoor environment during the day; the corresponding MAE and SD are only $2.28 \mathrm{bpm}$ and $1.52 \mathrm{bpm}$, respectively. Moreover, even if the user is wearing glasses, this has only a marginal impact on the heart-rate estimation effectiveness. Compared with an extant PC-based heart-rate estimation system, the proposed system is more accurate. The proposed system is simple to operate, requiring the user to adjust the position of the front camera so that it is aligned with his or her face, which is similar to looking at a mirror, and the heart rate can be estimated in only $3.6 \mathrm{~s}$. Hence, the proposed system demonstrates practical value.

\section{Acknowledgment}

This work was supported by the Ministry of Science and Technology under grant number MOST 103-2221-E-024-013.

\section{References}

[1] E.C. Kyriacou, C.S. Pattichis and M.S. Pattichis, An overview of recent health care support systems for eEmergency and mHealth applications. Proceedings of 31st Annual International Conference of the IEEE Engineering in Medicine and Biology Society (2009), pp. 1246-1249.

[2] M. Boulos, S. Wheeler, C. Tavares and R. Jones, How smartphones are changing the face of mobile and participatory healthcare: An overview, with example from eCAALYX. BioMedical Engineering OnLine 10, 24 (2011).

[3] W.L. Chen, T.H. Tsai, C.C. Huang, J.H. Chen and C.D. Kuo, Heart rate variability predicts short-term outcome for successfully resuscitated patients with out-of-hospital cardiac arrest. Resuscitation 10, 1114 (2009).

[4] J.K. Chiang, M. Koo, T.B. Kuo and C.H. Fu, Association between cardiovascular autonomic functions and time to death in patients with terminal hepatocellular carcinoma. Journal of Pain and Symptom Management 4, 673 (2010).

[5] T.G. Pickering, White coat hypertension. Current Opinion in Nephrology and Hypertension 2, 192 (1996). 
[6] A.N. Nicolaides, Diagnostic evaluation of patients with chronic venous insufficiency, Vascular Surgery, edited by R.B. Rutherford, WB Saunders Company (1989).

[7] R. Hull, W.G. van Aken, J. Hirsh, A.S. Gallus, G. Hoicka, A.G.G. Turpie, I. Walker and M. Gent, Impedance plethysmography using the occlusive cuff technique in the diagnosis of venous thrombosis. Circulation 1, 696 (1976).

[8] A.B. Hertzman, Photoelectric plethysmograph of the fingers and toes in man. Proceedings of the Society for Experimental Biology and Medicine (1937), p. 529.

[9] R.J. Whitney, The measurement of volume changes in human limbs. Journal of Applied Physiology 1, 1 (1953).

[10] M.Z. Poh, D.J. McDuffk and R.W. Picard, Non-contact, automated cardiac pulse measurements using video imaging and blind source separation. Optics Express 10, 10762 (2010).

[11] D.Y. Chen, J.J. Wang, K.Y. Lin, H.H. Chang, H.K. Wu, Y.S. Chen and S.Y. Lee, Image sensor-based heart rate evaluation from face reflectance using Hilbert-Huang transform. IEEE Sensors Journal 1, 618 (2015). 